\title{
Influencia de la pauta de dosificación sobre la adherencia terapéutica en jóvenes
}

\author{
Influence of dosage schedule on the therapeutic adherence in young \\ Influência do esquema de dosagem sobre a adesão em jovens
}

Rafael Manuel de Jesús Mex-Álvarez

Facultad de Ciencias Químico Biológicas, Universidad Autónoma de Campeche, México

rafammex@uacam.mx

Patricia Margarita Garma-Quen

Facultad de Ciencias Químico Biológicas, Universidad Autónoma de Campeche, México

pamgarma@uacam.mx

\section{Resumen}

La adherencia terapéutica implica el cumplimiento, de parte del paciente, de las indicaciones del terapeuta para mejorar su estado de salud; la adherencia a largo plazo es importante para prevenir complicaciones de salud y evaluar la efectividad del régimen terapéutico, dado que las enfermedades crónicas se han convertido en un problema creciente de salud pública porque su falta de control provoca problemas psicosociales y económicos como depresión, costos elevados para el sistema de salud, un aumento en los gastos para el paciente y mayores ausencias en el trabajo. Por ello, el objetivo de este trabajo fue estudiar los factores que influyen en la adherencia terapéutica en jóvenes estudiantes universitarios por medio de experimentos sencillos de administración de placebo; el número de individuos participantes fue 114 voluntarios (53 hombres y 61 mujeres) con rango de edad de 19-25 años (edad promedio 21 años); se emplearon dos métodos para medir la adherencia terapéutica: uno basado en la medición del número de comprimidos consumidos y el otro en un cuestionario de autoevaluación. Los resultados del estudio demuestran que el género y el número de dosis son factores importantes que 
influyen sobre la adherencia al tratamiento; las mujeres se apegaron mejor al tratamiento aunque ambos sexos disminuyeron el cumplimiento cuando se aumentó la frecuencia de administración de dosis (porcentaje cumplimiento del tratamiento: $83 \%$ en hombres y 88 $\%$ en mujeres con tres comprimidos, y $54 \%$ en hombres y $65 \%$ en mujeres con 7 comprimidos al día). Además, se demostró que existen discrepancias entre los resultados de ambos métodos empleados, los hombres tendieron a mentir más respecto al cumplimiento del tratamiento (porcentaje cumplimiento: hombres, método subjetivo $60 \%$ y método objetivo 54\%; mujeres, método subjetivo $68 \%$ y método objetivo $65 \%$ ).

Palabras clave: adherencia farmacoterapéutica, salud pública, pacientes jóvenes.

\section{Abstract}

Therapeutic adherence implies the compliance, on the part of the patient, advised by the therapist to improve their state of health; long-term adherence is important to prevent health complications and assess the effectiveness of the therapeutic regimen, given that chronic diseases have become a growing problem of public health because their lack of control leads to psychosocial and economic problems such as depression, high costs for health system, an increase in costs for the patient and major absences at work. By this, the objective of this work was study the factors that influence in the adhesion therapeutic in young students University by means of experiments simple of administration of placebo; the number of individuals involved was 114 volunteers (53 males and 61 females) with age range of 19-25 years (age average age 21); two methods for measuring the adherence were used: one based on the measurement of the number of consumed pills and the other in a self-assessment questionnaire. The results of the study show that the gender and number of dose are important factors influencing adherence to treatment; women stuck better treatment though both sexes decreased compliance when the frequency of dose was increased (treatment compliance percentage: $83 \%$ in men and $88 \%$ in women with three tablets, and 54\% for males and $65 \%$ in women with 7 capsules per day). In addition, it was demonstrated that there are discrepancies between the results of both methods, men tended to lie more on the treatment compliance (percentage compliance: men, subjective method 
$60 \%$ and $54 \%$ objective method; women, subjective method $68 \%$ and $65 \%$ objective method).

Key Words: pharmaco-therapeutic adhesion, public health, young patients.

\section{Resumo}

A adesão implica o cumprimento do paciente, as indicações terapeuta para melhorar a sua saúde; A longo prazo, a adesão é importante para prevenir complicações de saúde e avaliar a eficácia do regime terapêutico, uma vez que as doenças crônicas tornaram-se um problema crescente de saúde pública devido a sua falta de controle faz com que problemas psicossociais e econômicas, como depressão, custos elevados para o sistema de saúde, o aumento dos custos para o paciente e aumentou as ausências do trabalho. Portanto, o objetivo deste trabalho foi estudar os fatores que influenciam a adesão em jovens universitários através de administração simples experimentos placebo; o número de indivíduos envolvidos foi de 114 voluntários (53 homens e 61 mulheres), com faixa etária 19-25 anos (idade média de 21 anos); foram utilizados dois métodos para medir a adesão: uma baseada na medição do número de comprimidos consumidos e outro em um questionário de auto-avaliação. Os resultados do estudo mostram que o género e o número de doses são factores importantes que influenciam a aderência ao tratamento; mulheres sejam respeitados melhor ao tratamento, embora ambos os sexos redução da complacência quando a frequência de administração (taxa de cumprimento do tratamento foi aumentado: $83 \%$ em homens e $88 \%$ em mulheres com três comprimidos, e $54 \%$ em homens e $65 \%$ em mulheres com 7 comprimidos por dia). Além disso, foi mostrado que existem discrepâncias entre os resultados dos dois métodos empregados, os homens tendem a mentir mais com relação à adesão ao tratamento (percentagem de conformidade: homens, subjetiva $60 \%$ e método método de destino $54 \%$ de mulheres, método subjetivo $68 \%$ e método segmentar $65 \%)$.

Palavras-chave: aderência farmacoterapia, de saúde pública, pacientes jovens.

Fecha recepción: Agosto $2016 \quad$ Fecha aceptación: Enero 2017 


\section{Introducción}

Se entiende por adherencia terapéutica al proceso por el cual el paciente sigue adecuadamente las indicaciones del terapeuta y cumple con las prescripciones recibidas, e implica el grado conductual del paciente para corresponder a las recomendaciones acordadas con el profesional sanitario para la toma de la medicación (Salinas Cruz y Nava Galán, 2012. Alba Dios et al., 2015). La adherencia terapéutica es de suma importancia para obtener resultados favorables en el tratamiento de enfermedades porque si no se apega al régimen terapéutico de nada serviría contar con métodos de diagnóstico avanzados y medicamentos altamente eficaces (Salinas Cruz y Nava Galán, 2012). El término de cumplimiento terapéutico implica el grado de actuación de un paciente de acuerdo con la dosis, la pauta posológica y el plazo prescrito; los resultados clínicos de un tratamiento farmacológico dependen tanto del modo de cómo tomen su medicación los pacientes como del tiempo que lo hagan (Dilla et al., 2009). La falta de adhesión al tratamiento terapéutico tiene una prevalencia alta y afecta a la práctica clínica especialmente en los tratamientos a largo plazo, como la farmacoterapia de pacientes con enfermedades crónicas, por ejemplo, la diabetes, la hipertensión o el cáncer (Chacón et al., 2015. García Cedillo y Morales Antúnez, 2015).

La Organización Mundial de la Salud considera como tema prioritario de salud pública la falta de adherencia al tratamiento terapéutico debido a que conlleva consecuencias negativas clínicas y económicas: fracasos en la farmacoterapia, un aumento de los costos sanitarios por incremento en las tasas de hospitalización y un mayor índice de mortalidad (Conthe y Tejerina, 2007. Arrivillaga Quintero, 2010. Perrín Santolaya et al., 2012). El origen del incumplimiento terapéutico es multifactorial y se debe a razones relacionadas con el paciente, quien es el foco central del problema; además el cumplimiento terapéutico depende del conjunto de elementos implicados en el proceso: la enfermedad, el personal sanitario y el sistema de salud. Entre los factores debidos al paciente destacan la edad, el entorno cultural y social, el nivel de educación e incluso la personalidad (Martín Alfonso y Grau Abalo, 2004; Ortiz Parada, 2008; Serrano et al., 2014). 
Diversas situaciones conducen a una falta de adherencia al tratamiento farmacológico, a veces es una falta no intencionada debido a olvidos o confusión, pero también el paciente puede dejar de tomar la medicación voluntariamente; por ejemplo, cuando percibe una mejoría a mitad del tratamiento, cuando existe temor a reacciones adversas o cree que la medicación es innecesaria o excesiva (Soria Trujano et al., 2012; Salcedo Barajas et al., 2014). La adherencia terapéutica deficiente en el tratamiento de enfermedades crónicas es un problema de salud con un alarmante crecimiento, especialmente en jóvenes que por sus actividades diarias tienden a olvidar el cumplimiento de la medicación. Se estima en promedio que $50 \%$ de los pacientes no se adhieren al tratamiento en los países industrializados; es de interés evaluar la adherencia al tratamiento farmacológico porque un desapego al mismo provoca ineficacia de la terapéutica, incremento en los costos de la atención en salud y problemas de índole personal, familiar y social (Ortiz y Ortiz, 2005; Ortiz Parada, 2008). Los problemas relacionados con la adherencia terapéutica se presentan tanto por exceso o defecto en el seguimiento de la recomendación y sucede en cualquier momento (antes, inicio, durante o fin) del tratamiento; aún más, en terapias complejas los pacientes pueden adherirse a ciertos aspectos de la prescripción pero no a todos, o algunos en determinadas circunstancias pero en otras no (Salvador Ortiz, 2004; Ortiz y Ortiz, 2005).

El problema de la falta de adhesión a la terapia es complejo, independiente de si es intencionada o no, y para su abordaje es necesario identificar cuáles son los factores que afectan a determinado grupo y poder diseñar estrategias individuales para corregirlo y evitar sus consecuencias negativas (Martín Alfonso y Grau Abalo, 2004; Serrano et al., 2014). En enfermedades crónicas que inician desde la adolescencia o la juventud, la resistencia al cumplimiento terapéutico también involucra factores psicosociales, por ejemplo, en casos de diabetes tipo 1 hay manifestaciones de rebeldía además de otros estados emocionales vinculados al proceso de aceptación de la enfermedad (Ortiz y Ortiz, 2005). Uno de los factores que se considera causal del incumplimiento terapéutico es la complejidad de las pautas posológicas, un mayor número de fármacos y múltiples dosis predisponen a la falta de apego; considerando esto el objetivo del presente trabajo fue conocer cómo afecta la complejidad de las pautas de medicación en la adherencia al tratamiento en jóvenes estudiantes universitarios. Para ello y con el propósito de simplificar 
los factores influyentes en la falta de adherencia en este trabajo se estudió el fenómeno en pacientes sanos, al obviar los factores dependientes de la enfermedad y el sistema de salud.

\section{Metodología}

Se diseñó un estudio observacional descriptivo cuyo objetivo era determinar los factores que influyen en la adherencia terapéutica en jóvenes estudiantes universitarios por medio de experimentos sencillos de administración de placebo (comprimidos de azúcar de diferentes colores: blanco, amarillo, azul y rojo); el número de individuos participantes fue 114 voluntarios: 53 hombres y 61 mujeres con rango de edad de 19-25 años (edad promedio 21 años). Se simularon cuatro regímenes farmacoterapéuticos, cada uno con duración de 14 días: a) el primero fue la administración de un comprimido cada 8h (tres tomas diarias); b) el segundo con la administración de un comprimido A cada $8 \mathrm{~h}$ y un comprimido B cada 24h (cuatro comprimidos/día); c) el tercero con la administración de un comprimido A cada 8h y un comprimido B cada $12 \mathrm{~h}$ (5 comprimidos/día); d) el cuarto consistió en la administración de un comprimido A cada $6 \mathrm{~h}$ y un comprimido $\mathrm{B}$ cada $8 \mathrm{~h}$ (7 comprimidos/día). Al inicio del experimento se instruyó a los participantes sobre el uso del pastillero y la importancia de la adherencia terapéutica, finalmente se suministraron los comprimidos requeridos durante cada periodo y se indicaron las pautas posológicas de cada uno.

\section{Condiciones de aplicación}

No se admitieron en el estudio a personas que cumplían con uno o más criterios de exclusión (estaban en un régimen terapéutico en el periodo de estudio, que superaban el rango de edad o declararan consumir alcohol o fumar regularmente) o que se negaron a la participación, previa solicitud de su consentimiento, y se eligió al siguiente participante voluntario. A cada participante se le hizo una pequeña entrevista para recolectar los datos sociodemográficos del estudio (edad, sexo, estado civil, licenciatura, grado y situación

familiar: vive solo o con algún allegado); además se le preguntó si padece alguna enfermedad. 


\section{Evaluación subjetiva del cumplimiento terapéutico}

Al inicio de la terapia se dispensaron los placebos a manera de dosis unitarias en cada uno de los pastilleros distribuidos por día; para medir la adherencia terapéutica se emplearon dos métodos: el primero subjetivo mediante la resolución de un cuestionario que constaba de dos partes: 1) preguntas abiertas para conocer las situaciones que se presentaban durante el tratamiento que dificultaban o impedían el cumplimiento y 2) una tabla calendario (tabla 1) para que el participante señalara su cumplimiento, a manera de autoevaluación.

Tabla 1. Ejemplo de la tabla calendario proporcionada a cada participante para el autocontrol de su adhesión terapéutica.

\begin{tabular}{|c|c|c|c|c|c|c|c|c|c|}
\hline \multicolumn{10}{|c|}{ Código de Paciente } \\
\hline \multicolumn{3}{|c|}{ Medicamento Amarillo } & \multicolumn{7}{|c|}{ Tomar un comprimido cada 8 horas } \\
\hline & Hora & Día 1 & Día 2 & Día 3 & Día 4 & Día 5 & Día 6 & Día 7 & Observaciones \\
\hline \multirow{3}{*}{$\begin{array}{c}\text { Semana } \\
1\end{array}$} & $6: 00$ & & & & & & & & \\
\hline & $14: 00$ & & & & & & & & \\
\hline & 22:00 & & & & & & & & \\
\hline \multirow{3}{*}{$\begin{array}{c}\text { Semana } \\
2\end{array}$} & $6: 00$ & & & & & & & & \\
\hline & $14: 00$ & & & & & & & & \\
\hline & 22:00 & & & & & & & & \\
\hline
\end{tabular}

Ponga en cada recuadro una $\sqrt{ }$ si tomó la medicación o una $X$ si faltó a ella, escriba en el cuadro de observaciones cualquier dificultad que tuviera para cumplir o las causas del incumplimiento del tratamiento. Fuente: elaboración personal.

\section{Medición objetiva de la adherencia terapéutica}

A posteriori, como método de verificación se usó el recuento de comprimidos para medir el grado de adherencia terapéutica; durante el transcurso del experimento y sin previo aviso, los días 3, 7, 9, 12 y 14 se contabilizó el número de formas farmacéuticas existentes en el pastillero. El porcentaje de cumplimiento se calculó dividiendo el número total de comprimidos presumiblemente consumidos por el número total de comprimidos que debería haber consumido y multiplicando por 100. 


\section{Análisis de datos}

Los datos obtenidos se capturaron, almacenaron y procesaron con el programa Microsoft Office Excel 2016 ®; primero los datos de las encuestas se tabularon y posteriormente se realizó un análisis descriptivo por un análisis exploratorio gráfico y por los cálculos de los porcentajes y promedios intragrupales. Se realizó un análisis descriptivo de las variables con un intervalo de confianza de $95 \%$, seguido de un análisis para comprobar la relación de la variable independiente principal (adherencia terapéutica) entre los valores obtenidos en los cuestionarios y los conteos de acuerdo a las variable sociodemográficas sexo, estado civil y situación familiar. Para ello se usaron las pruebas estadísticas de $\mathrm{t}$ de Student y Ji cuadrada $(\mathrm{p}<0.05)$ y se empleó el programa estadísticos SPSS 22.0 para la realización del análisis.

\section{Resultados}

Los resultados del cumplimiento de las pautas posológicas durante el experimento se muestran en las figuras 1 y 2 , donde se puede observar que existe una tendencia (líneas punteadas) a desapegarse al tratamiento farmacológico a través del tiempo del tratamiento y de acuerdo a la complejidad del régimen posológico, es decir, con el aumento en la frecuencia de la dosis y el número de medicamentos indicados; en este estudio no se incluyó el factor tiempo de duración del tratamiento farmacológico (todos fueron de 14 días), por lo que se recomienda estudiarlo para incluir más factores al análisis; aunque cada experimento se realizó en el mismo grupo de personas se dieron 7 días entre cada régimen posológico como periodo de descanso. En este trabajo se reportan los resultados obtenidos de los pacientes que concluyeron los cuatro tratamientos, inicialmente el grupo era mayor, pero por diversas causas 25 voluntarios decidieron desertar del estudio. 
Figura 1. Porcentaje de estudiantes masculinos que cumplieron con la pauta posológica a través del experimento $(\mathrm{T} x=$ tratamiento, $\mathrm{C}=$ comprimido, las líneas representan la tendencia polinominal en cada Tx.)

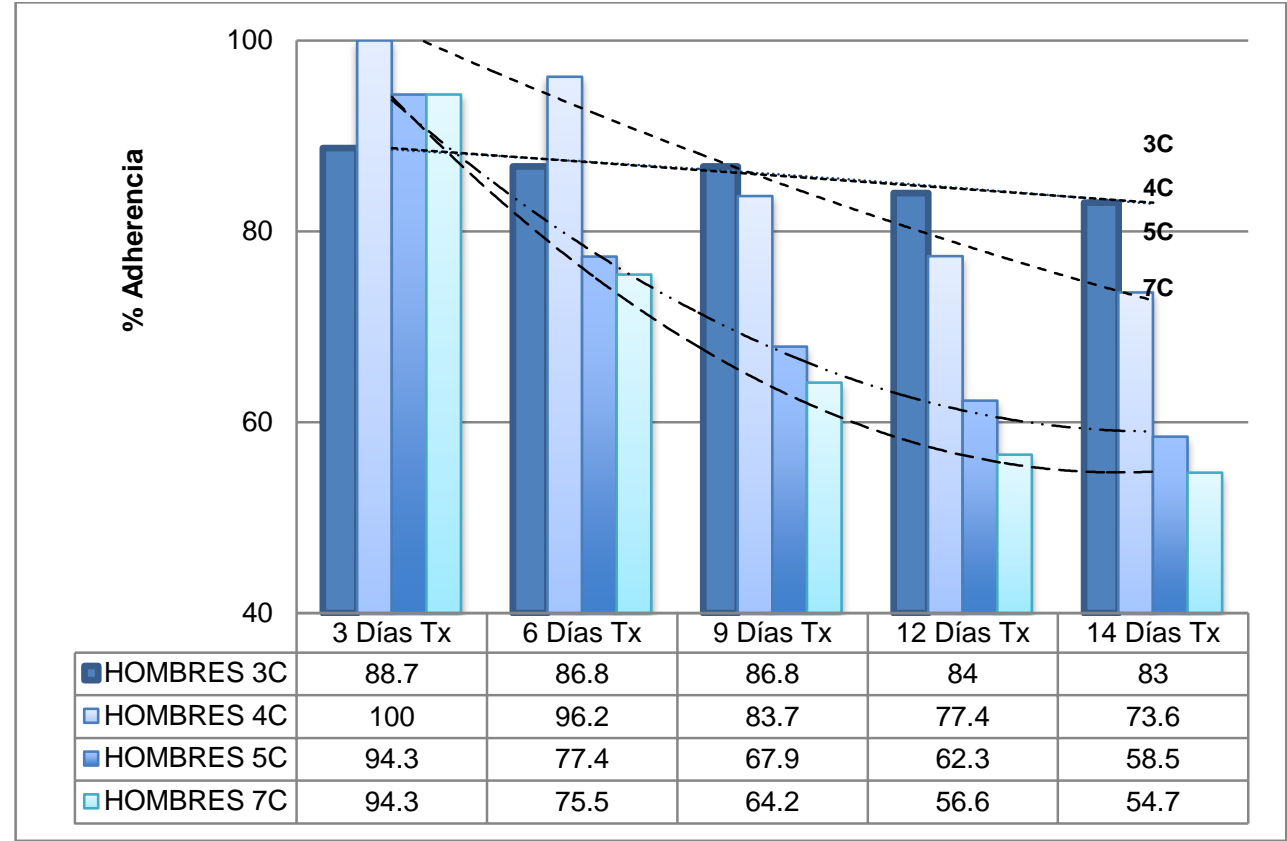

Fuente: cuestionario.

La falta de cumplimiento del régimen posológico aumenta en ambos grupos (hombres y mujeres) de acuerdo al número diario de comprimidos que tenían que consumir; cuando tenían que tomar 3 comprimidos se mantuvo casi constante el porcentaje de incumplimiento (menor pendiente de la línea de tendencia), la falta de adhesión aumentó más pronunciadamente con los regímenes multidosis de 5 y 7 comprimidos. 
Figura 2. Porcentaje de estudiantes femeninos que cumplieron con la pauta posológica a través del tiempo del experimento $(\mathrm{Tx}=$ tratamiento, $\mathrm{C}=$ comprimido, las líneas representan la tendencia polinominal en cada Tx.).

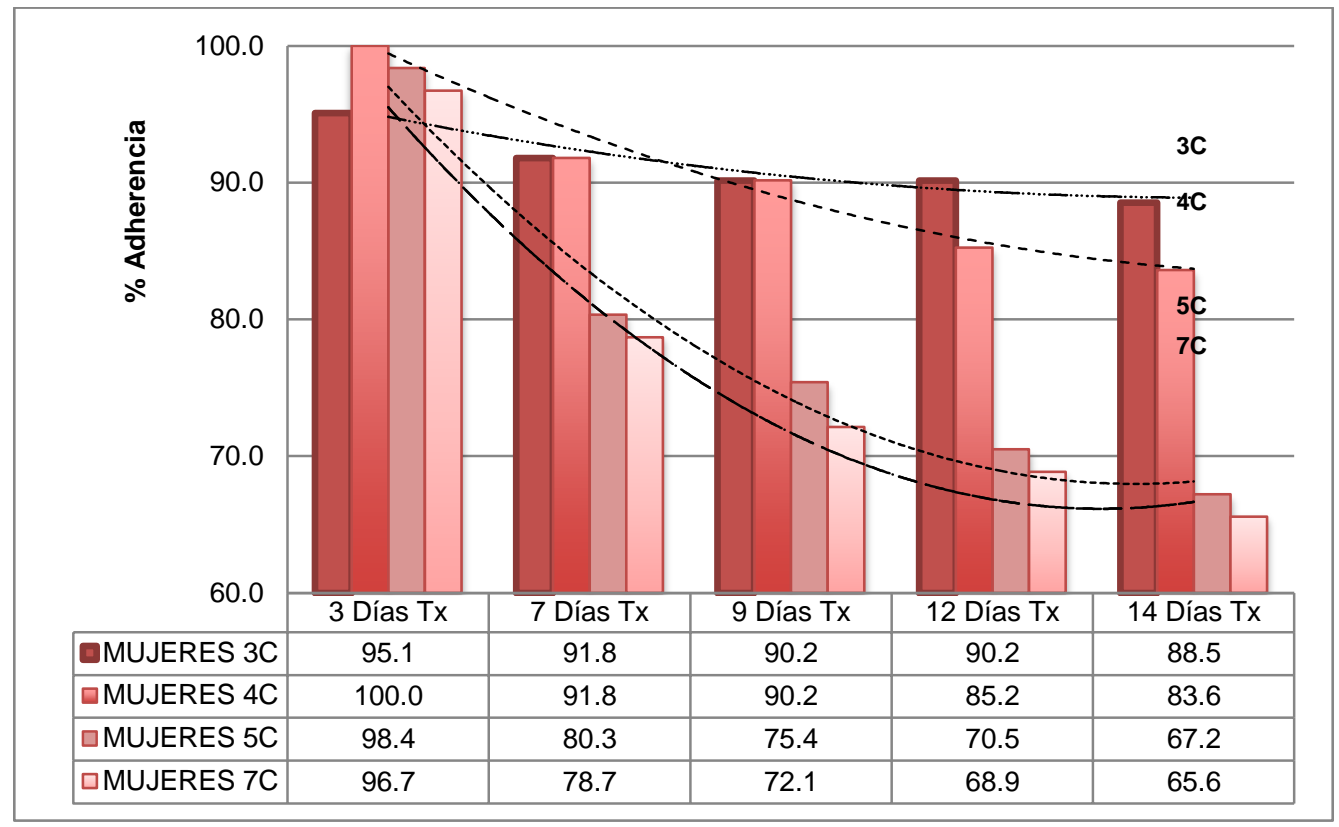

Fuente: cuestionario.

Sin embargo, al comparar los resultados mostrados en las figuras 1 y 2 se puede observar que el porcentaje de adherencia terapéutica es menor en hombres que en mujeres; en la figura 3 se grafican los porcentajes del incumplimiento del tratamiento al final de cada tratamiento y se puede observar que un mayor porcentaje de hombres no cumplió con los tratamientos (de 17.0 a $45.3 \%$ ) en comparación con las mujeres (de 11.5 a $34.4 \%$ ); sin embargo, la tendencia de aumento del porcentaje de incumplimiento (al aumentar el número de comprimidos para administrar) es semejante en ambos grupos. 
Figura 3. Porcentaje de incumplimiento de cada grupo de voluntarios al final de cada tratamiento (día 14 del experimento, $\mathrm{COM=}$ comprimidos).

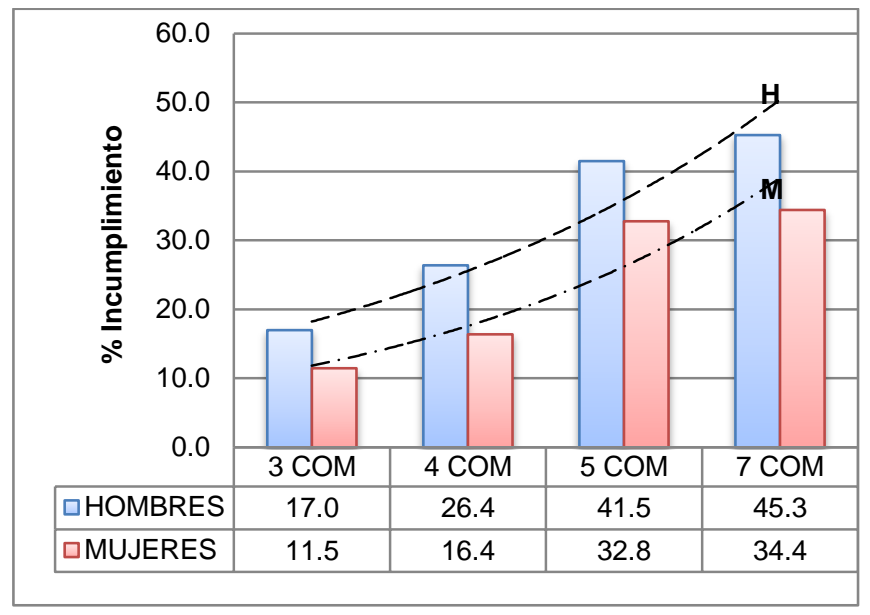

Fuente: cuestionario.

A pesar de que ambos géneros tendían a minimizar la falta de apego al tratamiento (como se observa en la figura 4), los hombres fueron menos confiables porque hubo un mayor porcentaje que en la autoevaluación (prueba subjetiva), donde reportaba que cumplía, pero en la revisión de los pastilleros se demostraba que no habían consumido el comprimido correspondiente.

Figura 4. Comparación del Porcentaje de adherencia subjetivo y objetivo de cada grupo al final del experimento (día 14 del estudio, $\mathrm{H}=$ Hombre, $\mathrm{M}=$ Mujer, $\mathrm{Sub}=$ subjetivo, $\mathrm{OBJ}=$ Objetivo, $\mathrm{COM}=$ Comprimidos).

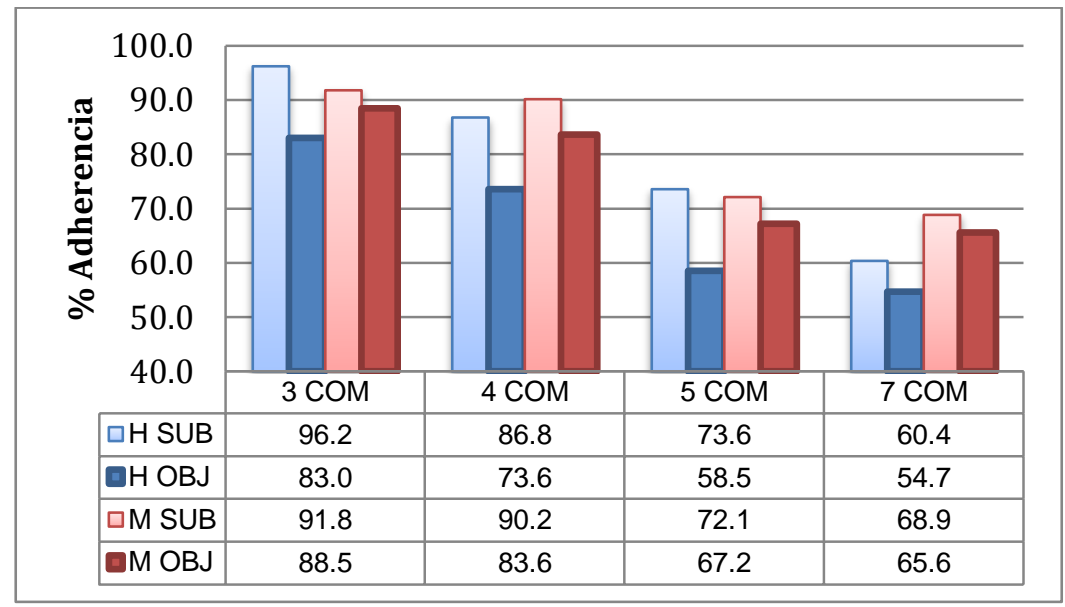

Fuente: cuestionario. 
En la figura 5 se grafican las diferencias porcentuales de la adhesión a las pautas posológicas, es decir, entre la diferencia del cumplimiento que los estudiantes reportaban contra el cumplimiento real; esta diferencia es mayor en los hombres (promedio $11.8 \%$ ) que en mujeres (promedio $4.5 \%$ ).

Figura 5. Diferencia porcentual del cumplimiento reportado (subjetivo) con el cumplimiento determinado (objetivo) de cada grupo al final del experimento $(\mathrm{COM}=$ comprimido).

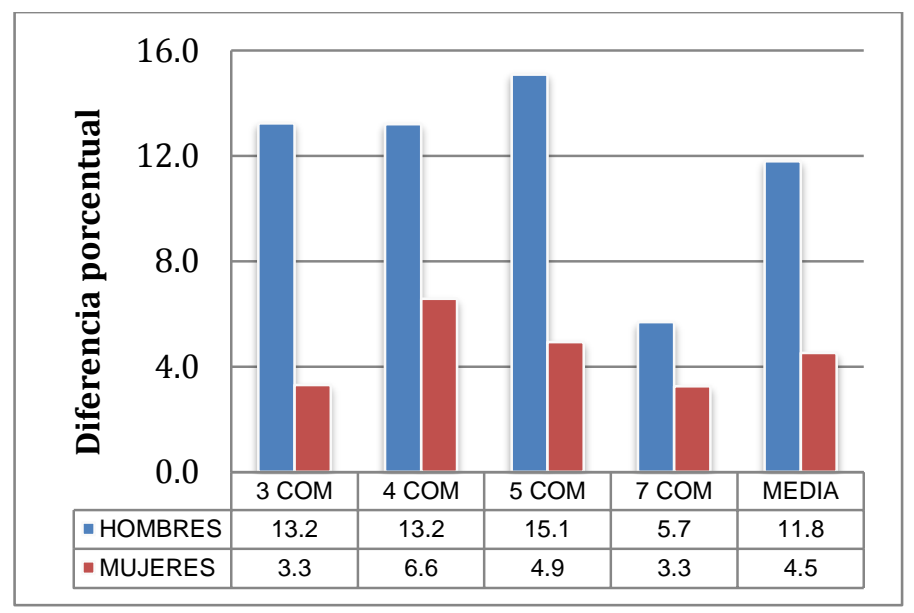

Fuente: cuestionario.

El principal instrumento que emplearon los participantes para recordar la ingesta del comprimido fue la alarma del teléfono celular, 41 (77 \%) de los hombres y $54(88 \%)$ de las mujeres empleó este método. Al investigar por qué los demás estudiantes no lo habían empleado, se tuvo que 4 hombres y 5 mujeres no tenían celular o el modelo disponible no permitía la configuración de diversas alarmas o etiquetar la actividad que alertan.

A pesar de que se les instruyó sobre el uso del pastillero y que cada participante contaba con uno para la organización de la administración, solamente 24 hombres (45.3) y 58 mujeres $(95.1 \%)$ llevaron siempre el pastillero, como se constató durante las revisiones (20 revisiones en total, 5 en cada tratamiento y 4 tratamientos totales); de ellos, 13 hombres y 18 mujeres (54.2 y $31.0 \%$ respecto al total de los participantes que llevaban pastillero) no lo usaban correctamente. El uso del pastillero fuera de su casa se dificulta más a los hombres (39 participantes, $73.6 \%$ ) que a las mujeres (12 participantes, $19.7 \%$ ), principalmente porque los hombres generalmente no acostumbran llevar cosas con ellos o 
por falta de espacio cuando salen a pasear, pues durante las clases lo transportaban en su mochilas pero generalmente los fines de semana los varones no llevan bolsos y por ello reportaban que se les complicaba transportarlos (en la bolsa del pantalón o en la mano e incluso lo olvidaban en la guantera de su automóvil).

La adherencia a un régimen terapéutico requiere de compromiso y atención a los horarios establecidos y adaptación de las actividades del tratamiento a las que se realizan de forma cotidiana; la mayoría de los encuestados (46 hombres, $86.8 \%$, y 59 mujeres, $96.7 \%$ ) notificó que era muy estresante seguir el régimen de dosificación, especialmente después del mes de implementado (tercer y cuarto tratamiento). Las diversas actividades que realizan los jóvenes complican adherirse al tratamiento, principalmente los fines de semana en los que adoptan una actitud de descanso y relajación; 48 hombres (90.6 \%) y 49 mujeres (80.3\%) respondió que es difícil integrar las actividades terapéuticas en su itinerario habitual.

Al inicio del estudio, 49 hombres $(92.5 \%)$ y 35 mujeres $(57.4 \%)$ tuvieron dificultad para organizar la administración de los comprimidos y, aunque no se cuantificó, se observó que solamente un número mediano de estudiantes cumplía con el tratamiento pues varios participantes masticaban los comprimidos (no los ingerían con agua, como se instruyó al inicio del estudio), porque cuando recordaban que era la hora de ingestión no habían previsto portar agua para ingerirlo y otros lo consumían con refrescos o junto con las comidas.

Finalmente, un factor positivo que estimulaba el cumplimiento del tratamiento fue la participación activa y el acompañamiento del personal sanitario a los participantes. Tanto hombres como mujeres manifestaron que la motivación (48 hombres y 61 mujeres) y el interés mostrado por parte del personal sanitario (52 hombres y 61 mujeres) eran actitudes positivas que les estimulaba a cumplir con la posología; también mencionaron ( 52 hombres y 61 mujeres) que la vigilancia no represiva (ausencia de reproche, castigo o estigmatización) de parte del personal sanitario representaba el compromiso de ellos con el 
paciente y que motivaba a corresponder de manera recíproca al esfuerzo mostrado por el personal sanitario.

\section{Discusión}

El problema de la adherencia terapéutica es grave y diversos estudios estiman que más de la mitad de pacientes con tratamientos crónicos no siguen de manera adecuada las indicaciones; el problema puede deberse a diferentes causas dependientes del paciente, de la enfermedad, del medicamento y del personal de salud (Merayo Alonso et al., 2008; Alba Dios et al., 2015; Martín Alfonso et al., 2015). Hay factores psicosociales que influyen de modo negativo en la adhesión al régimen terapéutico, entre ellos destaca la percepción del estado de salud y de la enfermedad que ocasionan ansiedad y depresión (Soria Trujano Trujano et al., 2011; Urzúa et al., 2011; Serrano et al., 2014).

Algunos métodos para la evaluación del cumplimiento farmacoterapéutico se basan en cuestionarios que el paciente tiene que resolver, por ello siempre que se puede debe corroborarse con algún método de referencia, por ejemplo, en el caso de la diabetes se contrasta con el control de glicemia y en el caso de pacientes hipertensos con los valores de presión arterial (Díaz Romero et al., 2004; Jiménez Herrera, 2014; Maldonado Reyes et al., 2016). Esto es importante porque el fracaso terapéutico puede deberse a la falta de compromiso y cumplimiento de parte del paciente y si no se contempla que el fracaso en el logro de las metas propuestas en el plan farmacéutico pueda deberse al incumplimiento del régimen posológico entonces se malgastan recursos al demandar otros fármacos, más estudios de laboratorio y consultas o tiempo de hospitalización por un mal control de la enfermedad (Merayo Alonso et al., 2008; Dilla et al., 2009; Vidal Corominas y Chamblás García, 2014; Chacón et al., 2015).

Según Orueta Sánchez (2005), los métodos de detección de la adherencia se dividen en directos (por determinación del fármaco o sus metabolitos en plasma u orina) e indirectos (por interrogatorios, cuestionarios, control del proceso y recuento de comprimidos); cada uno presenta sus ventajas y desventajas, el principal problema de los cuestionarios de autoevaluación consiste en que el paciente tiende a mentir para evitar ser regañado, por ello 
los profesionales deben procurar generar un ambiente adecuado al no culpabilizar al paciente y tratar de profundizar la relación profesional-paciente y que las intervenciones deben integrarse en la práctica clínica diaria. Este punto es crítico para la toma de decisiones debido a que el fracaso terapéutico puede deberse a la falta de adherencia, en cuyo caso se debe controlar el cumplimiento, en cambio si el paciente es cumplidor y no se obtienen los resultados deseados se debe realizar una revisión a la farmacoterapia para optimizarla. Estos señalamientos coinciden con los de Jiménez Herrera (2014), quien señala que los diversos métodos de medición de la adherencia permiten agrupar el comportamiento del paciente pues el proceso de adherencia es dinámico y complejo por diversos factores involucrados, por lo que se requiere de un mayor consenso e investigación en los diferentes aspectos involucrados en la adherencia terapéutica. Bolaños Cardozo (2014) señala la importancia de una evaluación más objetiva y concreta para promover una intervención terapéutica basada en modelos integrales.

En este estudio se observó que al aumentar el número de comprimidos disminuía la adherencia a la medicación; en este sentido coincide con la investigación de Sontakke et al. (2015), quienes con un esquema promedio de tres a seis medicamentos tomados diariamente reportaron que $70 \%$ de los pacientes no se adherieron al tratamiento. Una cifra similar señala Jiménez Herrera (2014), en su estudio aproximadamente $50 \%$ de los pacientes se adhieren al tratamiento farmacológico; entre los factores relacionados con el cumplimiento, según Jiménez Herrera, destacan las características asociadas a la persona (como el sexo, el conocimiento y las actitudes) y las características del régimen terapéutico (número de medicamentos, la dosis, la frecuencia, la duración y los esquemas terapéuticos complejos).

El papel del profesional de salud debe ser motivar y acompañar al paciente en su tratamiento para que se adhiera al mismo y cumpla con las indicaciones que los profesionales de salud le brindan, más que un papel inquisitivo que rompería la comunicación sincera con el paciente el farmacéutico y todos los demás profesionales de la salud deben mostrar empatía con el paciente para que reconozca que no cumple con el tratamiento pero comprenda las complicaciones que conlleva así como los aspectos negativos de su conducta para que adquiera un compromiso que permita el éxito del 
tratamiento (Quiroga Garza, 2012; Jiménez Herrera, 2014; Serrano et al., 2014; García Cedillo y Morales Antúnez, 2015).

El estilo de vida más demandante complica el cumplimiento del tratamiento terapéutico, las múltiples actividades que tienen los estudiantes favorecen el olvido y el desapego a sus nuevas obligaciones; en particular manifestaron que los fines de semana se complicaba más seguir las indicaciones por sus tareas en equipo y sus responsabilidades familiares, sociales, religiosas y culturales. También el cuestionamiento social genera sentimientos de culpa o vergüenza que provocaban no tomar los medicamentos en público y si no encontraban algún espacio o tiempo oportuno omitían la dosis correspondiente. Rand (2002) señala un tipo de no adhesión terapéutica denominado adherencia errática que se debe a dosis olvidadas por diversas causas, entra las que destacan los días festivos o vacaciones, también los horarios apretados o complejos. Aunque existe una buena intención por cumplir con el tratamiento existe complicaciones por la complejidad de su estilo de vida, que interfiere con la adherencia y no prioriza el adecuado control de la enfermedad con la farmacoterapia. De igual manera, las personas con horarios laborales complicados o estilos de vida caóticos presentan dificultad para establecer hábitos de un nuevo régimen de medicación; en ese mismo sentido, entre semana se presenta una buena adherencia pero los fines de semana o días festivos hay una disrupción en la rutina farmacoterapéutica. Este mismo fenómeno se observó en los estudiantes universitarios, pues se tiene horarios de clases complicados y mixtos, actividades académicas y tareas, actividades extracurriculares (artes y deportes) además de las actividades sociales que influyen en una conducta de adherencia errática en los jóvenes estudiantes.

La correcta intervención del farmacéutico permite recordar y aumentar el cumplimiento por parte del paciente, algunos estudios muestran que el uso de la tecnología contemporánea ayuda a incrementar los niveles de cumplimiento (Quiroga Garza, 2012; García Cedillo y Morales Antúnez, 2015). En este estudio los estudiantes mejor adaptados a las nuevas tecnologías de comunicación las usan para recordar los horarios de la ingesta, como sucedió con el uso del teléfono celular donde programaban alarmas para que sepan cuándo tomarlo; algunos comentaron que han visto a familiares mayores que anotan cuándo tomar y para qué sirve cada medicamento en el empaque secundario (cajas) y que pensaban que era 
bueno para tener presente el uso adecuado del medicamento. Las nuevas generaciones tan adaptadas a la tecnología deben descubrir y valorar el uso de herramientas no tan modernas pero igualmente útiles, como el uso del pastillero que permite clasificar y controlar la administración de las dosis. Tal vez el uso de la tecnología sea más difundido e interviene de un modo no previsible tanto de modo negativo como positivo, este aspecto puede estudiarse en investigaciones posteriores para dilucidar mejor su efecto sobre la conducta del paciente respecto a su medicación. El personal de salud debe fijarse en las fortalezas personales del paciente y ayudarlo a interpretar las dificultades como un reto y no como una amenaza (Martín Alfonso y Grau Abalo, 2004; Marayo Alonso et al., 2008; Soria Trujano et al., 2011).

Conocer que los estudiantes varones se apegan menos al tratamiento debe conducir a un mayor monitoreo del cumplimiento de la terapia; el sentirse jóvenes y sanos actuó de modo negativo porque los pacientes que no se adhirieron reportaron que no fueron responsables al no percibir un peligro inminente o un prejuicio inmediato; este factor psicológico de seguridad afecta negativamente a las personas, si alguien ingiere una sustancia y muere es más factible que quienes experimentaron ese hecho no beban dicha sustancia, en cambio si se ingiere una sustancia cuya toxicidad es crónica y no se aprecia bien que es la causante de la muerte hay más posibilidad que no se perciba un riesgo, como sucede en el caso de los fumadores que saben que puede causar cáncer pero como se puede fumar por un periodo largo sin que se afecte la salud de un modo evidente, no reconocen el riesgo y no existe un cambio conductual a pesar de que tienen el conocimiento intelectual (Soria Trujano et al., 2012; Vidal Corominas y Chamblás García, 2014; Chacón et al., 2015).

También sucedió que los estudiantes no tenían precaución en colocar sus "medicamentos" en un lugar adecuado, y tres hombres y una mujer solicitaron que se les proporcionaran de nuevo los medicamentos porque algún familiar menor de edad (niños menores de seis años) había ingerido los placebos. Esto alerta sobre la necesidad de formar una cultura sanitaria de seguridad y concientización del buen almacenamiento y resguardo de los medicamentos, que no deben dejarse al alcance de los niños. El entorno familiar desempeña un papel importante en el cumplimiento de la farmacoterapia debido a que los familiares conscientes y comprometidos con la salud del paciente ayudan a vigilarlo, motivarlo y a que se organice 
para que siga las indicaciones y recomendaciones del personal de salud (Meraya Alonso et al., 2008; Soria Trujano et al., 2012; Alba Dios et al., 2015; Chacón et al., 2015).

Esto coincide con algunos investigadores que señalan que los estilos de vida ejercen un efecto importante en la salud del paciente y se debe integrar como parte de las medidas terapéuticas para el control de su enfermedad; el conjunto de hábitos de conducta del paciente influye en el seguimiento del régimen terapéutico, por eso debe investigarse qué pautas de conducta tiene y utilizarlas para el reforzamiento de conductas benéficas que favorezcan el tratamiento (Dilla et al., 2009; Martín Reyes, 2010; Urzúa et al., 2012; Chacón et al., 2015).

El posible diagnóstico de la evolución de su estado de salud o de la enfermedad afecta el cumplimiento del tratamiento, tanto si el paciente percibe que tomando o no la medicación estará sano (como en el caso de una enfermedad infecciosa leve) como si percibe que a pesar de que siga el tratamiento la enfermedad no desaparecerá (Martín Reyes, 2010; Soria Trujano et al., 2011; Alba Dios et al., 2015). Es conveniente formar al personal de salud con herramientas psicológicas que le permitan emplear recursos personológicos del paciente para favorecer la adhesión al tratamiento al elevar su compromiso, su carácter activo-participativo, su autodeterminación y responsabilidad; es decir, se debe evitar que el paciente genere estrés y frustraciones perjudiciales y favorecer actitudes positivas que incentiven el crecimiento personal (Merayo Alonso et al., 2008; Soria Trujano et al., 2011; Chacón et al., 2015). Algunos investigadores plantean que la familia es la primera red de apoyo social para el paciente, por su acción protectora ante las adversidades y sirve como un factor positivo de promoción de hábitos saludables y preservación de la salud; además, generalmente conduce al soporte afectivo para el paciente (Soria Trujano et al., 2012; Vidal Corominas y Chamblás García, 2014; García Cedillo y Morales Antúnez, 2015). 


\section{Conclusión}

Los resultados obtenidos en este estudio sugieren que el género y la complejidad del régimen terapéutico son dos factores que influyen en la adherencia terapéutica porque las mujeres se apegaron mejor al tratamiento que los hombres, aunque ambos sexos mostraron un menor cumplimiento cuando aumentó la multidosis. Comparando los dos métodos de evaluación de la adherencia terapéutica se observó que los pacientes masculinos tienden a mentir más que los pacientes femeninos, porque en el cuestionario de autoevaluación reportaron un mayor cumplimiento contrastado con el cumplimiento real estimado por el método de recuento de comprimidos. El estudiar a estudiantes sanos simplifica el análisis del proceso de adherencia terapéutica, pues no están implicados aspectos psicológicos y sociales relacionados con una enfermedad específica; entre los factores identificados en este trabajo que dificultan el seguimiento terapéutico están el tener un itinerario complejo con múltiples actividades diarias de diversa índole (académicas, artísticas, deportivas y socioculturales) y la falta de un medio eficaz como recordatorio. Un porcentaje significativo de estudiantes programaron su teléfono celular para controlar el itinerario de medicación y con alarmas para avisar que debían administrarse la medicación. La mayoría de los estudiantes no supo usar el pastillero, este hecho constituye una oportunidad de mejora que debe considerarse en la educación sanitaria y concientización de pacientes

jóvenes porque el pastillero es un recurso útil para una mejor organización y cumplimiento de las pautas posológicas. 


\section{Bibliografía}

Alba Dios M.A., Pérula de Torres C.J., Pérula de Torres L.A., Pulido Ortega L., Ortega Ruiz R., Jiménez García C. (2015). Adherencia terapéutica y calidad de vida relacionada con la salud en pacientes crónicos polimedicados de más de 65 años. Med Fam Andal 16 (2): 111-124.

Arrivillaga-Quintero, M. (2010). Análisis de las barreras para la adherencia terapéutica en mujeres colombianas con VIH / SIDA: cuestión de derechos de salud. Salud Pública de México 52(4): 350-356.

Bolaños Cardozo (2014). Validación de un instrumento para valorar la adherencia de pacientes al tratamiento hipertensivo. Nova 12 (21): 115-119.

Chacón J., Sandoval D., Muñoz R., Romero T. (2015). Evaluación del control de la presión arterial y la adherencia terapéutica en hipertensos seguidos en el programa de salud cardiovascular (PSCV). Asociación con características clínicas, socioeconómicas y psicosociales. Revista Chilena de Cardiología 34(1): 18-27.

Conthe P., Tejerina F. (2007). Adhesión al tratamiento y calidad de vida en los pacientes con insuficiencia cardiaca. Rev Esp Cardiol Supl 7: 57-66.

Díaz Romero R.M., Mendoza Flores M.E., Belmont Padilla J. (2004). Validación de un instrumento para evaluar la adherencia terapéutica en diabéticas durante el embarazo. Perinatol Reprod Hum 18(4), 217-224.

Dilla T., Valladares A., Lizán L., Sacristán J.A. (2009). Adherencia y persistencia terapéutica: causa, consecuencias y estrategia de mejor. Aten Primaria 41 (6): 342348.

García Cedillo I, Morales Antúnez B.V. (2015). Eficacia de la entrevista motivacional para promover la adherencia terapéutica en pacientes con diabetes mellitus tipo 2 . Universitas Psychologica 14 (2), 511-522. 
Jiménez Herrera L. (2014). Adherencia terapéutica y oportunidades de mejora del estado salud-enfermedad. Rev Costarr Salud Pública 23 (1): 68-74.

Jiménez Herrera L. (2014). Adherencia terapéutica y oportunidades de mejora del estado salud-enfermedad. Rev. Costarr. Salud Pública 23:68-74.cd

Maldonado-Reyes F. J., Vázquez-Martínez V. H., Loera-Morales J., Ortega-Padrón M. (2016). Prevalencia de adherencia terapéutica en pacientes hipertensos con el uso del cuestionario Martín Bayarre-Grau. Aten Fam 23(2): 48-52.

Martín Alfonso L., Bayarre Vea H., Corugedo Rodríguez M.C., Vento Iznaga F., La Rosa Matos Y., Orbay Araña M.C. (2015). Adherencia al tratamiento en hipertensos atendidos en áreas de salud de tres provincias cubanas. Revista Cubana de Salud Pública 41 (1), 33-45.

Martín Alfonso L., Grau Abalo J.A. (2004). La investigación de la adherencia terapéutica como un problema de la psicología de la salud. Psicología y Salud 14 (001): 89-99.

Martínez Reyes F. (2010). Adherencia a la terapéutica farmacológica en pacientes hipertensos del Hospital "José Carrasco Arteaga". Cuenca 2007. Revista de la Facultad de Ciencias Médicas. 29 (31): 82-94

Merayo Alonso L.A., Cano García F.J., Rodríguez Franco L. (2008). Motivación para el cambio como predictor de la adherencia terapéutica en el dolor crónico. Apuntes de Psicología 26 (2): 331-339.

Ortiz M., Ortiz E. (2005). Adherencia al tratamiento en adolescentes diabéticos tipo 1 chilenos: una aproximación psicológica. Rev. Méd. Chile 133: 307-313.

Ortiz Parada M. (2008). Estabilidad de la adherencia al tratamiento en una muestra de adolescentes diabéticos tipo 1. Ter Psicol 26(1): 71-80. 
Orueta Sánchez R. (2005). Estrategias para mejorar la adherencia terapéutica en patológicas crónicas. Información terapéutica del Sistema Nacional de Salud 29 (2): 40-48.

Perrín Santolaya R., Fernández-Pacheco García Valdecasas M., Arteche Eguizabal L., Gema Pérez I., Muñoz Muñoz N., Ibarra Barrueta O., Callejón Callejón G. (2012). Adherencia subóptima al tratamiento en la esclerosis múltiple. Farm Hosp 36 (3): $124-129$.

Quiroga Garza A. (2012). Intervención telefónica para promover la adherencia terapéutica con síntomas de ansiedad y depresión. Enseñanza e Investigación en Psicología 17 (2), 387-403.

Rand C.S. (2002). Adherence to Asthma Therapy in Preschool Child. Allergy 57 (Suppl. 74): $48-57$.

Salcedo Barajas A., Gómez Ochoa A.M. (2014). Grados de riesgo para la adherencia terapéutica en personas con hipertensión arterial. Av. Enferm. XXXII (1): 33-43.

Salinas Cruz E., Nava Galán M.G. (2012). Adherencia terapéutica. Enf Neurol 11(2): 102104.

Salvador Ortiz M. (2004). Factores psicológicos y sociales asociados a la adherencia al tratamiento en adolescentes diabéticos tipo 1. Psykhe 13(1): 21-31.

Serrano M.J., Vives M., Mateu C., Vicens C., Molina R., Puebla-Guedea M., Gili M. (2014). Adherencia terapéutica en pacientes depresivos de atención primaria: un estudio longitudinal. Actas Esp Psiquiatr 42 (3): 91-98.

Soria Trujano R., Ávila López D.I., Vega Valero Z., Nava Quiroz C. (2012). Estrés familiar y adherencia terapéutica en pacientes con enfermedades crónicas. Alternativas en Psicología XVI (26): 78-84. 
Soria Trujano R., Vega Valero Z., Nava Quiroz C., Saavedra Vázquez K. (2011). Interacción médico-paciente y su relación con el control del padecimiento en enfermos crónicos. Liber 17 (10): 223-230.

Urzúa M. A., Marmolejo C. A., Barr D. C. (2012). Validación de una escala para evaluar factores vinculados a la adherencia terapéutica en pacientes oncológicos. Univ Psychol 11(2): 587-598.

Vidal Corominas D.J., Chamblás García, I. (2014). Alianza terapéutica y adherencia a tratamientos de drogodependencia: un estudio en usuarios de centros de atención de la intercomuna. Revista Perspectivas 25: 143-166.

Sontakke S., Jadhav M., Pimpalkhute S., Jaiswal K., Bajait C. (2015). Evaluation of Adherence to Therapy in Patients of Type 2 Diabetes Mellitus. Journal of Young Pharmacists 7 (4): 462-469. 